\title{
Schedule Risk Analysis of ICT Infrastructure Projects
}

\author{
Malaya Kumar Nayak \\ Research Scholar, \\ Department of Computer Science \& Applications, \\ Utkal University, \\ Bhubaneswar, INDIA
}

\author{
Dr Sanghamitra Mohanty \\ Professor and Head, \\ Department of Computer Science \& Applications, \\ Utkal University, \\ Bhubaneswar, INDIA
}

\begin{abstract}
Scheduling a project is an inexact process in that it tries to predict the future. An ICT infrastructure project is a combination of interrelated activities that must be performed in a certain order of completion. To cut development costs and meet tight deadlines in ICT software Infrastructure Projects, managers need to understand some key doubts about the standard scheduling methods and how to use a schedule risk analysis to provide information crucial to an ICT Infrastructure Project's success before they embark on their ICT Infrastructure Project. This study deals with the benefit of conducting schedule risk analysis on an ICT Infrastructure Project. It is shown that such risk analysis has the potential to provide key information for ICT Infrastructure project managers in advance so that risk mitigation plans can be developed and implemented in time.
\end{abstract}

\section{General Terms}

Software Engineering, Requirements/Specifications, Project and People Management

\section{Keywords}

Risk planning, ICT, Scheduling Risk, Project planning, Simulation

\section{INTRODUCTION}

Risk management, which is an iterative process, in international ICT project management involves growing concerns these days. The risk management process comprises all the activities required to identify the risk that might have a potential impact on the ICT project. The project schedule is the core of the project planning. It is used by managers to commit people to the project and show the organization how the work will be performed. To cut development costs and meet tight deadlines in short-staffed software projects, managers must optimize the project schedule. To identify the risk involvement, various tools and techniques have been discovered, such as documentation review, information gathering, check list analysis, assumption analysis, diagramming techniques, and so on. Scheduling a project is extremely difficult as the time needed to complete a project activity is hard to estimate. Process simulation is an important technique to evaluate the impact of proposed changes [12] in the process.

System dynamics simulations have been around for years. Some of them address the problem of project staffing [1] and show how to use process simulation to support project managers in scheduling [19]. Many conservative project management skills have already been used to minimize the risk for ICT projects, but the important concern of project managers is to have an efficient schedule that ensures the completion of target overheads right on time. However it is concluded that the majority of ICT projects overrun their schedules most of the time. For example, a real-time software development recently added three months' duration between monthly timetable appraisals. But it has also been seen that sometimes timetable slippage arises in small increments but builds up over time, resulting in substantial time delays. Such types of issues are important concerns for project managers and leaders [2].

\section{ICT INFRASTRUCTURE PROJECT RISK ASSESSMENT METHODOLOGY}

A general understanding of the ICT Infrastructure project risk assessment methodology aids in the discussion of the application of ICT Infrastructure project risk analysis. The ICT Infrastructure project risk assessment is performed in two basic steps: a qualitative analysis followed by a quantitative analysis. The identification of potential risks requires a systematic review of the entire ICT Infrastructure project during which the technical, cost, and schedule risks are evaluated using ICT Infrastructure Project-specific risk rating criteria [7, 8, 9]. The ICT Infrastructure project risk assessment can be done by understanding and signifying the ICT project schedule's slip, which normally happens because of at least four issues that are normally coupled with ICT project risk analysis $[4,22]$ :

- Scheduling risk analysis is complex. In the process of software ICT project development it is very obvious that risk scheduling is one of the most significant disciplines that cannot be mastered by anyone who normally has project risk scheduling responsibilities.

- The traditional process of complicated protocols followed for risk scheduling, normally known to users as deployment of judgment, constraints, resources, datebook, and activity durations, is not often clear to the project managers Most project schedules, even those produced by experienced schedulers, need to be debugged before they can be assumed professionally proficient.

- Another most frustrating issue is that both client and project owner will often be adamant about adhering to a very unrealistic time limit. The project scheduler is often not permitted to produce a schedule that can be accomplished with the resources at hand. Professionalism in project scheduling would argue against many of the schedules forced on projects today.

- Normal risk schedules are designed using singlepoint (deterministic) approximations of activity durations. When the uncertainty of future durations is taken into account, the duration of a schedule path 
is likely to differ from that computed by scheduling and by deploying special scheduling software.

The qualitative assessments are then translated into numerical values that are used to develop the input data distributions, which in turn are fed into the quantitative model.

\section{POTENTIAL RISK OF SCHEDULE DELAY}

Before reviewing an activity, the analyst defines the potential risks based on the features of the activity. Schedule, cost, and technical risks cannot be considered independently, and cost and technical risks often influence the schedule risks [6].

Risk factor rating criteria are used to guide the qualitative analysis of each activity, which results in a risk rating of "high," "medium," or "low" for each activity. The qualitative results and any specialized quantitative data are used to develop the uncertainty distributions for each activity in the ICT infrastructure project risk model. Simulation models using tools such as Crystal Ball produce cumulative probability distribution functions (PDFs) that describe the confidence level given to the achievement of the ICT infrastructure project performance. Before analysing the risk factor rating criteria, there are many unsolved risk scheduling questions such as:

- Can these risk factors be predicted for specific ICT projects with some grade of precision?

- Can the reasons for ICT project risk be signified before they become serious difficulties, which would probably enhance the project management's capability to preclude their occurrence by taking effective risk lessening steps?

- Is it feasible to estimate the risk factors why projects often overrun their initial schedules?

ICT project risk analysis highlights head-on the fact that it is not known how long the activities will take. Even if an action has been taken before in a prior development, there is no guarantee that it will take the same amount of time in a new project. Nor is there any guarantee that amending it for the situations (for example, range, intricacy) of the new project will be precise. The situation will be different in the new project, with different resources and/or different efficiency to be expected. And, most prominently, risks in the work that occurred in the former project may not be accurately replicated in the new project, or risks that did not occur in that prior project may occur in the new one. In fact, it is generally not possible to estimate with certainty how extensive each activity will be or which risks will occur. Duration estimates are just that: estimates. The activities will occur in the future and 'there are no facts about the future' [20]. Even with the complete nonexistence of management or customer interference and the use of the best risk estimation techniques, the real-life action durations will diverge, sometimes dramatically, from those planned and included in the schedule [16].

\section{UNCERTAINTIES OF ICT INFRASTRUCTURE PROJECT SCHEDULING}

ICT Infrastructure project managers need to understand some uncertainties about the standard ICT Infrastructure project scheduling and how to use a schedule risk analysis to provide information crucial to an ICT Infrastructure Project's success before they embark on an ICT Infrastructure project [6]. The ICT Infrastructure project duration calculated is accurate only if everything goes according to plan. In many cases, the completion dates are unrealistically optimistic and highly likely to be overrun, even if the schedule logic and duration estimates are accurately implemented. The path identified as the risky path using traditional CPM techniques may not be the one that will be most likely to delay the ICT Infrastructure project and which may need management attention [20].

The contingency or uncertainties are normally analysed using Crystal Ball software, an add-in to the Microsoft Excel format of the cost estimate and schedule. Monte Carlo simulations can be conducted by applying the risk factors (enumerated as probability density functions) to the suitable estimated cost and schedule requirements identified by the ICT risk scheduling. Uncertainties are estimated by considering only the moderate and high level risks identified for each alternative (i.e., low-level risks are typically not considered, but remain within the risk schedule to serve chronological rationale as well as to support follow-on risk studies as the project and risks evolve).

For the cost estimate, the uncertainties are calculated as the difference between the expenditure forecast and the base cost approximation. Each option-specific uncertainty is then allocated on a works feature level based on the dollarweighted relative risk of each feature as quantified by Monte Carlo simulation. Standard deviation is used as the featurespecific measure of risk for contingency allocation purposes. This approach results in a relatively larger portion of all the project feature cost uncertainties being allocated to features with relatively higher estimated cost uncertainty. For schedule uncertainties analysis, the option schedule uncertainties are calculated as the difference between the option duration forecast and the base schedule duration. These uncertainties are then used to calculate the time value of money impact of project delays. The resulting time value of money, or added risk escalation, is then added to the uncertainties amount to reflect the standard for presenting the 'total project cost' for the fully funded project amount. The schedule contingency is analysed only on the basis of each option and not allocated to specific tasks. Based on Cost Engineering DX guidance, only critical path and near critical path tasks are considered to be uncertain for the purposes of contingency analysis [11, 13, $14]$.

\section{SIMULATION OF THE ICT INFRASTRUCTURE PROJECT SCHEDULE}

Once the activities' duration ranges and distributions have been determined, the schedule risk analysis can determine how risky the entire ICT Infrastructure project schedule is. The most common method of determining schedule overrun risk is to simulate the ICT Infrastructure project by solving (or iterating) it hundreds or thousands of times on the computer [3].

The risk model utilizes the risk register as its basis within the Excel format. The risk analyst and the project manager must determine the best modeling method that provides adequate cost and risk analysis model output for the project. The result is a customized model specifically related to the project. The final product must present contingencies in the desired format for the total project cost estimate. For example, risk analyses can be performed for each contract (assuming there are several contracts), for each project feature, or for the total project base cost [15]. 
In developing the cost risk model template, two common approaches are recommended. The approaches are dependent upon what the project manager prefers as the outcome when establishing the uncertainties. For example, if just a single project or contract is planned, a single uncertainty value may be desired for efficient planning for risk management. The project manager may prefer a contingency developed for each project feature. If several projects or contracts are planned, the project manager may desire a separate contingency for each project. These issues should be resolved before preparation of the cost and risk analysis model. Typical approaches currently used are as follows:

1) Risk event based: The model is developed to reflect the completed risk register. In a sense, the risk register becomes the model. In that way, the risk estimation techniques' outputs directly reflect the established risk events on the risk register. This output supports the proposed system well when related to document traceability, risk management, and follow-on risk studies. The cost and risk analysis output is risk based and typically presents the contingency for each project. This method can be obtained from Cost Engineering DX.

2) Cost based: This method might be used when several contracts are being studied within the programme and separate contract contingencies or feature contingencies are desired. The resulting model reflects the work breakdown structure (WBS) within the estimate(s). To pursue this avenue, the cost and risk analysis model is developed by downloading the most likely estimate into an Excel file at the chosen WBS level that best relates to the risk register. Once that is accomplished, the market study and the various risk events are referred to, creating the best and worst case estimates, again reflecting the chosen WBS. The cost and risk analysis output is cost based, because it reflects the WBS within the estimate template and is more intuitive for the cost engineer. The advantage is that the model output can better reflect multiple project features, contracts, or different funding accounts. The disadvantage is that it does not directly correlate to the risk events established and is less traceable to the risk register. If this alternative is chosen, the report must document how the cost and risk analysis outputs relate to the risk register event concerns.

\subsection{Simulation of an Activity Network} Representing an ICT Infrastructure Project

A typical real-life ICT Infrastructure project was considered for simulation experiment. Ten distinct activities were identified in the ICT Infrastructure Project. With respect to each activity, three parameters, namely 1) most optimistic effort, 2) most pessimistic effort, and 3) most likely effort, required to carry out each activity were established. These three time values provide a measure of the uncertainty associated with that activity $[5,18]$. The proposed model is as shown in Figure 1 below.

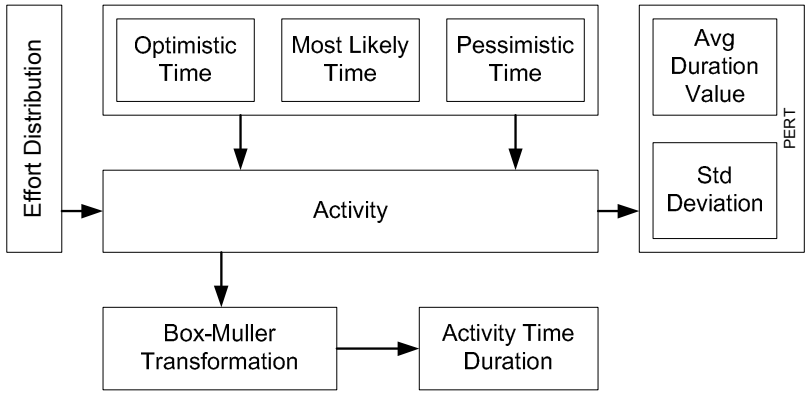

Figure 1: Proposed model

Optimistic Time $\left(t_{0}\right)$ : The optimistic time is the shortest possible time in which the activity can be finished.

Most Likely Time $\left(t_{1}\right)$ : The most likely time is the estimate of the normal amount of time the activity would take.

Pessimistic Time $\left(t_{p}\right)$ : The pessimistic time represents the longest time the activity could take if everything goes wrong.

The list of these activities is given in graphs. Each activity is specified by its starting node, finishing node, and duration. That is, $\mathrm{S}(\mathrm{k})$ is the node at which the $\mathrm{k}^{\text {th }}$ activity originates and $\mathrm{F}(\mathrm{k})$ is the node at which the $\mathrm{k}^{\text {th }}$ activity terminates, for $\mathrm{k}$ $=1,2, \ldots, N$. $\mu_{\mathrm{k}}$ (mean) and $\sigma_{\mathrm{k}}$ (standard deviation) are computed by assuming that the duration of each activity is given by a beta distribution that is,

The random variable $\mathrm{x}$ that is randomly distributed with shape $\alpha 1$ and $\alpha 2$

$\mathrm{x} @$ can be used $\beta(\alpha 1, \alpha 2)$

where

$$
\begin{aligned}
& \alpha 1=\mu((\mu(1-\mu) / \sigma)-1) \\
& \alpha 2=(1-\mu)((\mu(1-\mu) / \sigma)-1)
\end{aligned}
$$

Based on $t_{o}$ (optimistic), $t_{1}$ (most likely), and $t_{p}$ (pessimistic) estimates for each activity, the average values of the duration $\mu \mathrm{k}$ and standard deviation $\sigma \mathrm{k}$ are estimated by using the following formulas used in analytical procedure (PERT) $[10$, 21]:

$$
\mu_{k}=\left(t_{o}+4 t_{l}+t_{p}\right) / 6
$$

and

$$
\sigma_{k}=\left(\left(t_{p}-t_{o}\right) / 6\right) 2
$$

It was assumed that the duration of every activity (k) is distributed normally $\left(\mu_{\mathrm{k}}, \sigma_{\mathrm{k}}\right)$ with specified mean $\mu \mathrm{k}$ and standard deviation $\sigma \mathrm{k}$ as obtained from the above relations. The sample time durations of each activity (k) of the ICT Infrastructure project for different runs are drawn (using BoxMuller transformation) from a universe which follows a normal distribution $\left(\mu_{\mathrm{k}}, \sigma_{\mathrm{k}}\right)$. 
When the algorithm follows the Box-Muller (1958) transformation for estimating the time duration of each activity over the network, it allows uniformly distributed random variables to be transformed into a new set of random variables following a normal distribution. It starts with a group of pairs of two independent random numbers $\left(r_{p}, r_{p}+1\right)$ which come from a uniform distribution (in the range from 0 to 1). It estimates the time duration as a sample of $\mathbf{t}_{\boldsymbol{i}}$ where $\boldsymbol{i}$ varies from $\mathbf{1}$ to $\mathbf{N}_{\mathbf{s}}\left(\mathrm{N}_{\mathrm{s}}\right.$ : sample size $>50$, as per the definition of the Central Limit Theorem), where $\sigma_{\mathrm{k}}$ and $\boldsymbol{\mu}_{\mathrm{k}}$ are the standard deviation and mean, respectively, for the $\mathrm{k}^{\text {th }}$ activity. Implementing these $\mathbf{N}_{\mathrm{s}}$ samples, statistics for each activity with assumed standard deviation $\boldsymbol{\sigma}$ and mean $\boldsymbol{\mu}$ are estimated.

$$
\begin{aligned}
& t_{i}=s_{i}^{*} \sigma_{k}+\mu_{k} \\
& \mathrm{~s}=\operatorname{sqrt}(-2 \ln (\mathrm{r} 1)) \cos \left(2 \mathrm{pi}^{*} \mathrm{r} 2\right)
\end{aligned}
$$

Where $(\mathrm{r} 1, \mathrm{r} 2)$ is a pair of random numbers in the range $(0,1)$ and $\mathrm{s}$ is the desired sample from the standardized normal distribution. To estimate each time duration corresponding to a typical activity $\mathbf{k}$, a sample of $\mathbf{s}_{\mathrm{i}}$ is created by implementing a random number generator where $i$ varies from 1 to $\mathbf{N}_{\mathbf{s}}$. For each activity an independent set of $\sigma_{\mathrm{k}}$ and $\boldsymbol{\mu}_{\mathrm{k}}$ are assumed based on network dynamics.

\subsubsection{Algorithm}

1. Read input data for activity network corresponding to given ICT Infrastructure project consisting of $n$ activities (topologically ordered) and $\mathrm{m}$ nodes, representing effort distribution by activity and milestones, and number of simulation runs.

2. Initialize the simulation run counter.

3. Generate random input data (by invoking a random number generation routine) using the Box-Muller transformation to generate random samples which follow a normal probability distribution for the duration of the $\mathrm{k}^{\text {th }}$ activity from the population $\left(\mu_{\mathrm{k}}\right.$, $\left.\sigma_{\mathrm{k}}\right)$

4. Perform the forward pass through the activity (effort) network.

5. Perform the backward pass through the activity network.

6. Compute the risk index corresponding to each activity (effort).

7. Update the simulation run counter.

8. If the simulation run count is less than the number of simulation runs (as read in step 1), then go to Step 3 for the next simulation run.

9. Compute and print risk indices for each activity (effort).

10. Stop.

In Figure 2, we have taken 10 activities in the ICT project and represented the milestones i.e. A, B ....J and edges i.e. 1, 2, $3 . . .10$ of the ICT project.

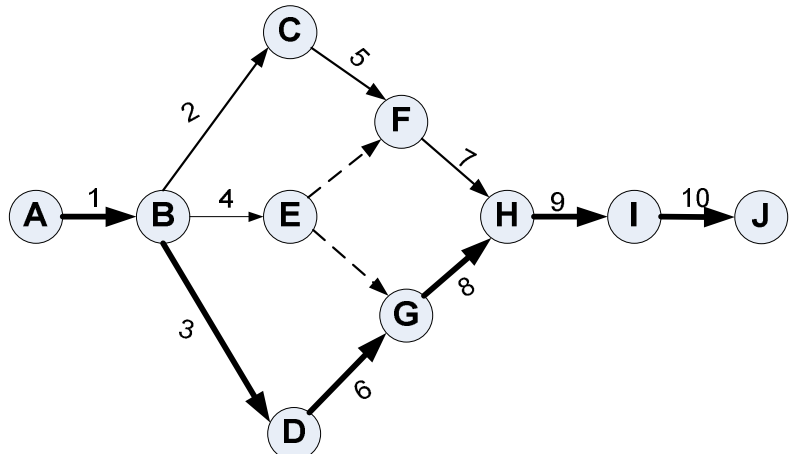

Figure 2: The network graph for ICT projects

To determine the entire pattern of possible completion dates for the project and its important milestones, the risk analyst iterates the project many times. At the end of every iteration, the completion dates for the total project and for any important milestones are collected and stored. The program also records which activities were on the risky path for that iteration.

At the end of the entire simulation, project completion and important milestone dates computed from all iterations are collected and arrayed in graphs showing the probability distribution, or relative frequency, of all possible dates.

Suppose that the risk analyst determines that 2,500 iterations will be sufficient for the accuracy needed. The result of that simulation is a cumulative likelihood distribution that represents the likelihood of the project being completed on or before each possible date. This distribution is shown in Figure 3:

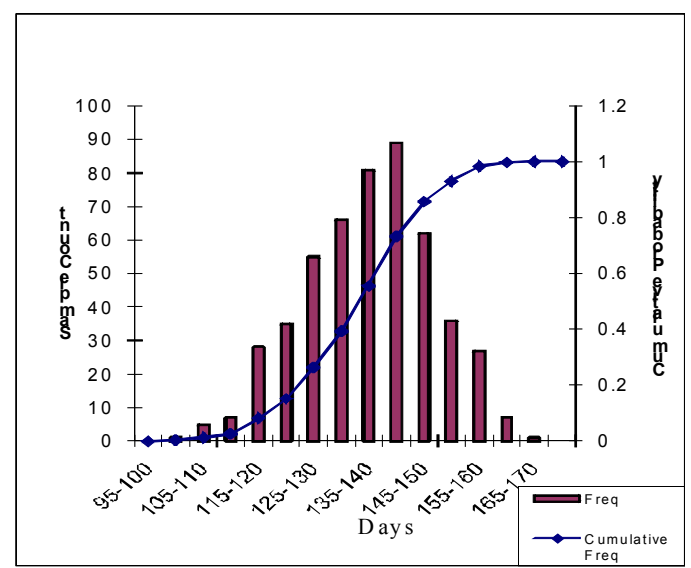

Figure 3: The distribution graph for ICT projects

Because each solution in a risk analysis must at least be feasible, it should not violate any resource limitations that exist. Each of iterations must be resource-leveled if any resource or resources are limited. The risk analysis software package should be able to level resources as it is, by iterating. This increases the run time substantially.

\section{CONCLUSION}

Accomplishments in managing ICT projects require a complete and realistic project schedule that represents the project plan. Project scheduling is one of the most important skills needed on the project team. However, projects often overrun their scheduled completion dates. Risk scheduling is a 
difficult discipline and individuals thrust into scheduling are not necessarily either suited to its demands or always effectively trained and supported. Also, in many instances, project schedulers are not permitted to develop realistic schedules since their management and the competitive customer-contractor environment lead to optimisticsometimes magical-schedules. These schedules sometimes lead to late delivery. One of the most important issues facing project scheduling is the inability to incorporate uncertainty of activity durations into the typical schedule. Activity duration estimates are of necessity based on assumptions that may prove untrue in fact, and the durations will differ from those estimated in many cases. The proposed scheme is again based on three parameters of time (optimistic, most likely, pessimistic), which actually encapsulate the highest probability of the estimated risk factors involved in ICT projects.

From the activity network given Figure 4, the risky activities were evaluated using the risk index value associated with an activity. The higher the risk index, the greater is the risk involved due to that activity. In the case considered here, Activities 7, 8, and 9 are the activities which need the attention of the risk analyst.

\section{RISK PROBABILITY GRAPH FOR ICT PROJECTS}

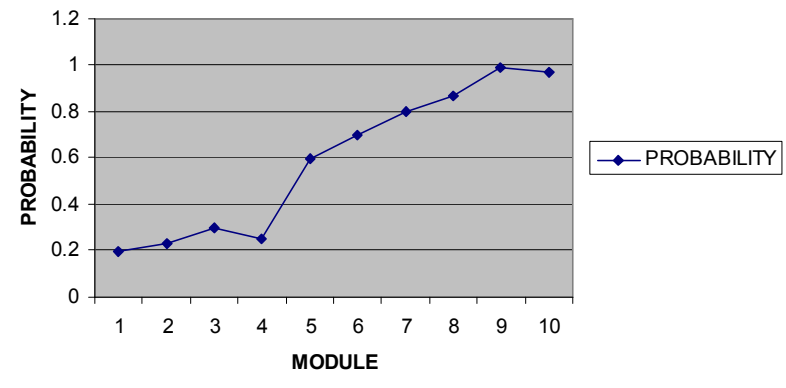

Figure 4: Risk probability graph for ICT projects

The risk analysis has the potential to provide key information for ICT infrastructure project managers in advance so that risk mitigation plans can be developed and implemented straight away. The experience with risk analysis also shows that developing the data and reviewing the results enables participants to understand and to manage their ICT infrastructure project better.

\section{ACKNOWLEDGMENTS}

We will quote special thankful to Dr. Rachna Soni, Associate Professor and Head, Department of Computer Science \& Applications, D.A.V. College for Girls, Yamunanagar, INDIA.

\section{REFERENCES}

[1] Abdel-Hamid, T. and Madnick, S., 1991. Software Project Dynamics, An Integrated Approach, Prentice Hall, Englewood Cliffs, New Jersey.

[2] AS/NZS 4360, 2004. Australian Standard: Risk Management, Standards Australia

[3] Banks, J., Carson, J. S., II, and Nelson, B. L., 1996. Discrete-Event System Simulation, second edition, Prentice Hall.
[4] Bannerman, P. L., 2008. 'Risk and risk management in software projects: A reassessment', Journal of Systems and Software, Vol. 81, No. 12, pp. 2118-2133.

[5] Bratley, P., Fox, B. L., and Schrage, L. E., 1987. A Guide to Simulation, second edition, Springer-Verlag.

[6] Deepti, V., Ramanamurthy, N., and Balasubramanian, K. U., 2004. 'Effective risk management: risk analysis using an enhanced FMEA technique'. Proc. of Annual Project Management Leadership Conference, India.

[7] Fishwick, P. A., 1995. Simulation Model Design and Execution: Building Digital Worlds, Prentice-Hall.

[8] Freund, J. E., 1992. Mathematical Statistics, fifth edition, Prentice-Hall.

[9] Gogg, T. and Mott, J., 1992. Improve Quality and Productivity with Simulation, Palos Verdes Pnsl., CA.

[10] Hogg, R. V., and Craig, A. T., 1995. Introduction to Mathematical Statistics, fifth edition, Prentice-Hall.

[11] Humphrey, W., 1997. Introduction to the Personal Software Process, Addison-Wesley.

[12] Kellner, M. I., Madachy, R. J., and Raffo, D. M., 1999. 'Software process simulation modeling: Why? What? How?' Journal of Systems and Software, Vol. 46, pp. 91-105.

[13] Kelley, J. E. and Walker, M. R., 1959. 'Critical-path planning and scheduling', Paper Presented at the Eastern Joint IRE-AIEE-ACM Computer Conference, ACM New York, NY, USA, December 1-3.

[14] Kerzner, H., 2009. Project Management: A Systems Approach to Planning, Scheduling, and Controlling, John Wiley and Sons.

[15] Kleijnen, J. P. C., 1987. Statistical Tools for Simulation Practitioners, Marcel Dekker, New York.

[16] Misra, S. C., Kumar V., and Kumar, U., 2005. 'Modeling strategic actor relationships to support risk analysis and control in software projects'. Proc. of the 2005 International Conference on Enterprise Information Systems, Miami, Florida, USA, May 25-28, pp. 288293.

[17] Montgomery, D. C., 1997. Design and Analysis of Experiments, third edition, John Wiley.

[18] Nelson, B. L., 1995. Stochastic Modeling: Analysis and Simulation, McGraw-Hill.

[19] Neumann, P. G., 1994. Computer-Related Risks, Addison-Wesley.

[20] Rodrigues, A., 1996. 'The role of system dynamics in project management', International Journal of Project Management, Vol. 14, No. 4, pp. 213-220.

[21] Suri, P. K. and Soni, R., 2007. 'Simulating the potential effect of risk management on project scheduling', Information Technology Journal, Vol. 6, No. 1, pp. 8-13.

[22] Tiwana, A., and Keil. M., 2004. 'The one-minute risk assessment tool', Communications of the ACM, Vol. 47, No. 11, pp. 73-77. 